\title{
El ciclo de aprendizaje experiencial en la enseñanza. Caso ESUMER-GIZ: Seminario sobre cooperación y relaciones comerciales con Alemania
}

\author{
EXPERIENTIAL LEARNING CYCLE IN TEACHING. ESUMER - GIZ CASE: SEMINAR ON COOPERATION AND \\ TRADE RELATIONS WITH GERMANY \\ O CICLO DE APRENDIZAGEM EXPERIENCIAL NO ENSINO.CASO ESUMER-GIZ: SEMINÁRIO SOBRE \\ COOPERAÇÃO E RELAÇÕES COMERCIAIS COM ALEMANHA
}

\author{
Lisbeth Duarte Herrera* / Lisbeth.duarte@esumer.edu.co \\ Andrea de la Hoz** / Andrea.delahoz@esumer.edu.co
}

\section{Resumen}

Este artículo es resultado del trabajo de campo realizado gracias al convenio de cooperación suscrito en el 2014 entre la Institución Universitaria ESUMER y la Agencia de Cooperación Alemana -GIZ1 , en el marco del programa "Global Business Exchange"2 .

Como producto de este programa, la universidad impartió el seminario de formación sobre cooperación y relaciones comerciales con Alemania dirigido a empresarios de pymes colombianas ${ }^{3}$ seleccionadas mediante convocatoria. En el artículo se presenta una reflexión sobre el método de aprendizaje utilizado en el seminario y los resultados obtenidos.

\section{Summary}

This article is the result of the fieldwork carried out thanks to the cooperation agreement signed in 2014 between ESUMER University and -GIZ, the German Cooperation Agency, under the "Global Business Exchange" program. As a result of this program, the university offered the training seminar on cooperation and trade relations with Germany addressed to entrepreneurs of Colombian SMEs selected by invitation. The article reflects on the learning method used in the seminar and the results obtained.

\section{Palabras Clave}

Aprendizaje, experiencia, metaplan, ciclo de aprendizaje.

\section{Key Words}

Learning, experience, metaplan, learning cycle

\footnotetext{
* Politóloga, de nacionalidad colombiana, Magister en Ciencias sociales con especialidad en Desarrollo del Colegio Mexiquense A.C, becada por el Conacyt de México. Docente e investigadora de ESUMER en la Facultad de Estudios Internacionales.

** Negociadora Internacional, de nacionalidad colombiana, Magister en marketing y comportamiento del consumidor de la Universidad de Granada, becada por el programa enlaza mundos. Docente e investigadora de ESUMER en la Facultad de Estudios Internacionales
}

1 Anteriormente conocida como GTZ

2 El programa Global Business Exchange inició en su primera versión a finales de 2012, cuenta con socios como: el Ministerio de Comercio Industria y Turismo (MinCit), La Agencia Presidencial de Cooperación Internacional (APC), ANDI, ACOPI, SENA, Universidad de los Andes e ICESI. En su segunda versión, la agencia alemana seleccionó nuevos socios adicionales a los que ya estaban vinculados entre los están: UNAB, Universidad del Norte y Esumer como socios en Bucaramanga, Barranquilla y Medellín, respectivamente.

3 De los sectores: energía renovable, alimentos e industria mecánica y eléctrica. 
Este artigo é o Resultado do trabalho de campo feito com ajuda do convênio de Cooperação subscrito no ano 2014 entre A Instituição Universitária ESUMER e a Agência de Cooperação Alemão-GIZ [1], no âmbito do Programa "Global Business Exchange" [2]. Como produto deste Programa, a Universidade deu O Seminário de Formação e Cooperação SOBRE Relações comerciais com Alemanha dirigidos aos Empresários de PYMES colombianas [3] selecionadas por meio de convocatória. No artigo se apresenta uma reflexão sobre o Método de Aprendizagem Utilizado no seminário y os Resultados conseguidos.
Aprendizagem, experiência, Metaplan, ciclo de aprendizagem.

\section{Introducción}

Alemania es un país donde la formación profesional se fundamenta en el modelo dual, en el cual el aprendizaje está dado a partir del diálogo entre el entorno académico y el laboral. Un modelo que desde luego, enriquece y dinamiza el conocimiento.

El modelo dual se basa en el aprendizaje experiencial donde existe una relación mutua entre experiencia y teoría. Se sustenta a su vez en las ideas de Dewey y Kolb para quienes el aprendizaje experiencial es un proceso que involucra distintas fases como son la experiencia concreta, la reflexión, la conceptualización y la aplicación.

Estos modelos de aprendizaje contrastan con el modelo de aprendizaje tradicional en donde el individuo es un sujeto pasivo, que sólo recibe información que eventualmente transformará en conocimiento.

La propuesta de aprendizaje experiencial se adapta a las nuevas demandas profesionales vinculadas con la formación y desarrollo de competencias y habilidades. Sin embargo, la experiencia por sí misma no genera aprendizaje si no está enmarcada en un proceso reflexivo mediante el cual se construye conocimiento a partir de la experiencia realizada (González, Marchueta, \& Vilche, 2013).

\section{Método de aprendizaje por experiencia}

Son muchos los estudios que se han preocupado por cómo se desarrolla el aprendizaje humano y de qué manera se puede definir. Este proceso puede abordarse desde distintas perspectivas, por lo que también existen distintas teorías del aprendizaje.

Una de ellas es la teoría del aprendizaje por experiencia que ofrece un enfoque diferente en el proceso de aprendizaje si se compara con otras teorías sobre las cuales se basa el modelo educativo tradicional.

Tomando como base esta teoría, Ataöv \& Ezgi Halilo囚lu Kahraman (2009) citando a Shotter's (2002) por ejemplo define el aprendizaje como aquel basado en la construcción de significado compartido a través de interacciones recursivas entre individuos. El aprendizaje se puede definir entonces como "el proceso en donde el conocimiento es creado por medio de la transformación de la experiencia" (Cliffs, 2006). 
La palabra clave es "experiencia", pues sus promotores enfatizan el rol central que la experiencia juega en cualquier proceso de aprendizaje, en particular, la forma como procesamos la experiencia y nuestra reflexión crítica sobre ella (Fowler, 2008).

Sus antecedentes se remontan al trabajo de Dewey (1938) quien partía del concepto de "experiencia más reflexión es igual a aprendizaje". En su trabajo defiende la idea de que es rol de los educadores impulsar prácticas que promuevan más experiencias favorables. Aunque recibió muchas críticas en la época, el concepto es ampliamente aceptado actualmente.

Metallidou \& Platsidou (2008) citando a Kolb (1984) por ejemplo, definen a la teoría del aprendizaje experiencial como "un proceso interactivo que involucra cuatro etapas diferentes que a su vez forman dos dimensiones bipolares de aprendizaje". Las cuatro modalidades de aprendizaje agrupadas en pares se definen como conceptualización abstracta vs experiencia concreta y experimentación activa vs observación reflexiva, en donde cada individuo utiliza alguna de estas modalidades en algún grado, pero al mismo tiempo, tiene preferencia por alguna de ellas.

(Nooghabi, Iravani, \& Fami, 2011) citando a Powell and Wells (2002) resumen las cuatro etapas del ciclo así:

- La experiencia concreta pone al alumno en una posición en donde aborda una situación concreta y la relaciona con su vida anterior usando los sentimientos más que la lógica.

- La observación reflexiva le permite al alumno hacer un escrutinio de ideas y reflexionar en la información desde diferentes puntos de vista.

- La conceptualización abstracta le permite al estudiante desarrollar generalizaciones o teorías para usar en la solución de problemas.

- La experimentación activa le permite al estudiante diagnosticar la solución o problema y usar habilidades conductuales para tomar acción.

El aprendizaje se adquiere, por lo tanto, a través de un ciclo de experiencia, reflexión y acción en donde reflexionar sobre experiencias conlleva a una mejora en la comprensión y una mejora en la comprensión lleva a una acción más efectiva (Abe, 2011).

El modelo argumenta que el alumno reflexiona sobre las experiencias de donde extrae significado y expresa ese significado a través del pensamiento, lenguaje, habla y acción. También expone un factor clave que es la conversación pues las conclusiones y el discurso se forman luego de que los estudiantes preguntan y cuestionan un nuevo significado acerca de sus experiencias y forman nuevas ideas gracias a las experiencias colectivas generadas en las conversaciones (Ataöv \& Ezgi Haliloglu Kahraman, 2009).

Muchos académicos apoyan este modelo de aprendizaje por la relación significativa entre aprendizaje y práctica. Por ejemplo Ataöv \& Ezgi Haliloglu Kahraman (2009) citando a Freire (1970) reivindica el rol esencial de la experiencia práctica de los individuos en el proceso de aprendizaje como una herramienta para empoderarlos. Además, define práctica como compuesta tanto por el aprendizaje como por el hacer.

El modelo o ciclo de aprendizaje por experiencia socializado por la agencia alemana, recoge los puntos esenciales de los trabajos antes mencionados y utiliza las siguientes fases o etapas entrelazadas entre sí. Las fases son: acción, publicación, procesamiento, generalización y aplicación.

En la fase de acción los participantes viven y experimentan una acción simulada asociada al tema que se va a tratar. En esta fase, se deben brindar instrucciones claras y precisas de acuerdo con el "mundo" que el facilitador recreará.

La fase de publicación es una etapa corta que alude al momento en el cual los participantes compartirán 
los resultados, estrategias y decisiones en plenaria, es una fase de socialización. La tercera fase es el procesamiento, entendido como el momento en el cual se toma distancia reflexiva y crítica frente al ejercicio simulado, en tanto se verbalizan las emociones y comparan las experiencias. La generalización es una etapa que busca tomar distancia frente a su propio caso y brindar resultados generales de sus experiencias buscando semejanzas entre el mundo simulado y el real.

La última fase, es la aplicación en ella se busca explorar estrategias acerca de cómo proceder en el futuro. Es importante tener en cuenta que las últimas tres fases, estas son, procesamiento, generalización y aplicación son orientadas por el facilitador a través de interrogantes y preguntas claves que guiarán la discusión. (ver esquema 1)

Esquema 1. Fases o etapas del ciclo de aprendizaje experiencial

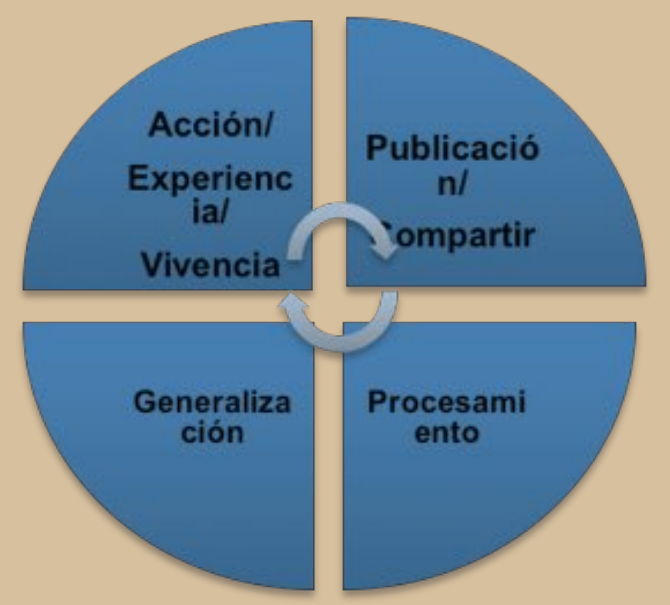

Fuente: Elaboración propia a partir del seminario recibido (Junio, 2014)

\section{Relato de la experiencia}

Como se señaló en la presentación de este artículo, la universidad se acercó al ciclo de aprendizaje por experiencia y técnica metaplan, a través del convenio de cooperación suscrito entre la Institución y la GIZ. Producto de este convenio, cuatro docentes de la institución recibieron un entrenamiento intensivo en junio de 2014, por parte de entrenadores senior del CEFE (Competency based economies formation of enterprise) ${ }^{4}$ en Mannheim Alemania, durante una estancia de 14 días. Cabe anotar que en este entrenamiento también participaron docentes de las otras instituciones socias.

El entrenamiento para entrenadores $(T O T)^{5}$ consistió en aproximarse y apropiarse del método de aprendizaje por experiencia y la técnica de expresión y visualización de ideas, metaplan, a través de un programa estructurado por los facilitadores del CEFE con macrolaboratorios; esto es, ejercicios prácticos donde los docentes participantes a partir de la vivencia, la experiencia y la lúdica, se iban apropiando de cada una de las fases que componen el método experiencial.

En el ciclo de aprendizaje por experiencia hay aspectos de forma y de fondo relevantes que se deben explicar con mayor profundidad en tanto entrarían a jugar como factores claves de riesgo o de éxito en la aplicación del método.

Uno de los aspectos de fondo es que el docente no es la única fuente del conocimiento, como comúnmente se conoce en el esquema de enseñanza tradicional, sino que es un facilitador del aprendizaje, lo cual a todas luces tiene una connotación y lectura distinta. Según la UNESCO (1993) el facilitador o tutor es un guía, orientador y apoyo en el aprendizaje de los alumnos, por consiguiente, es un agente de cambio con competencias para la innovación educativa.

En este modelo entonces la experiencia es la base y el estímulo para al aprendizaje en donde los alumnos aprenden activamente de manera holística en una construcción social y cultural influenciados por el contexto socio-emocional donde esto ocurre (Fowler, 2008).

4 Es un centro de formación que inició en 1983, como un conjunto integral de mecanismos de capacitación orientado a los métodos de aprendizaje por experiencia para desarrollar e incrementar competencias en administración de empresas, generación de ingresos y desarrollo económico sostenible. CEFE internacional tiene el apoyo y financiamiento del Ministerio alemán para la cooperación económica.

5 Training of trainers 
La nivelación de expectativas es otro aspecto, aparentemente sin trascendencia, pero en el fondo de gran relevancia en el método de aprendizaje experiencial, en tanto es el momento en el que los participantes plasman en forma escrita sus perspectivas, motivaciones $y$ expectativas frente al curso que se va a recibir. El objetivo es que al final del curso el estudiante de la mano del facilitador, regrese a las expectativas para evaluar si éstas fueron logradas o no durante el desarrollo del curso.

Otro de los aspectos de fondo a destacar dentro del método es: el contrato de aprendizaje, cuya finalidad es suscribir compromisos entre el o los facilitadores y los participantes. El contrato de aprendizaje es un acuerdo de voluntades que otorga un matiz de formalidad y da cuenta de la seriedad con la cual se debe asumir este proceso de aprendizaje entre las partes. El momento en el que se realiza el contrato de aprendizaje es justo después de la dinámica de nivelación de expectativas, pues el facilitador recalca que éstas se pueden lograr sólo si se imparten ciertas reglas y obligaciones.

La evaluación diaria al finalizar cada sesión de trabajo, es otro de los aspectos a destacar dentro del modelo experiencial, en tanto a través de una evaluación sencilla se busca indagar en el participante o estudiante acerca de: la relevancia del contenido, la construcción del conocimiento, la calidad de la presentación, la categoría aprendiendo de los demás y personalmente cómo se siente. Como un componente adicional dentro del proceso de formación está el de iniciar cada sesión de trabajo con una "reportería", es decir, en cada sesión los estudiantes o participantes por un espacio de 15 a 20 minutos y de manera creativa hacen un recuento o retrospectiva de lo visto en la sesión anterior. La idea es que los participantes se vayan rotando esta labor y que la creatividad prime en la realización de esta tarea.

Los contenidos a impartir en las clases se deben estructurar y ajustar de acuerdo con el ciclo o método de aprendizaje. Por esta razón, el facilitador debe previamente diseñar y preparar las actividades que los estudiantes desarrollarán en la fase de acción y posteriormente publicación, así como estructurar las preguntas de sensibilización y profundización que intervendrán en las fases de, procesamiento, generalización y aplicación.

Ahora bien, en lo que respecta a aspectos de forma, por supuesto, no menos importantes, sobresale la posición de las sillas en el aula de clase. Para efectos de recrear un ambiente de homogeneidad y de confianza, las sillas, en el método de aprendizaje experiencial, se ubican en forma de " $u$ " posición que brinda una sensación de apertura frente a las ideas y al conocimiento. Este aspecto puede ser un detalle meramente espacial que, ciertamente, rompe con la estructura tradicional y jerárquica de las aulas de clase habituales (véase figura 1). El fin último es generar la interacción a través de la conversación, pues ésta se convierte en un potencializador del aprendizaje en situaciones prácticas.

La ubicación de las sillas, además es un medio para incentivar un ambiente adecuado e impulsar la generación de emociones positivas en el aula. Se ha comprobado que la generación de emociones positivas juega un papel central en el éxito de un proceso de aprendizaje experiencial por varias razones tal como se evidencia en el trabajo de Abe (2011).

Primero, de acuerdo con la literatura existente sobre el tema, las emociones están relacionadas con la dinámica intrapersonal e interpersonal que en últimas facilita el proceso de aprendizaje experiencial. Segundo, las emociones positivas sirven como recursos sicológicos y sociales para hacer frente a los diferentes retos que se presentan en cada una de las etapas de desarrollo de la experiencia del curso. Por último las emociones positivas también pueden contribuir a fomentar la reflexión que ha sido identificada como una de las variables principales del proceso.

El número de estudiantes cobra gran valor en el ciclo de aprendizaje por experiencia, en tanto se trata de que los facilitadores interactúen con un grupo no muy numeroso pero tampoco muy pequeño, por lo cual una cifra que oscile entre los 15 y 20 participantes puede ser ideal para la aplicación del método. Igualmente, es importante que el facilitador conozca previamente el grupo meta u objetivo hacia el cual va dirigido el programa, con el fin 
de estructurar y ajustar con mayor precisión las actividades y contenidos a desarrollar. No es lo mismo estructurar un curso a un grupo de gerentes de grandes empresas, a empleados en el área de las ventas o incluso a docentes.

Ahora bien, dentro del ciclo de aprendizaje por experiencia, la técnica metaplan6, también conocida como técnica de moderación grupal, se destaca como una herramienta fundamental o factor clave del éxito en la aplicación correcta del método. De acuerdo con EPISE (2007) "el metaplan busca la interactividad y la motivación del participante" (pág. 3). En ese orden de ideas, "cuando decae el interés del participante el metaplan añade una ayuda en forma de animador o tutor que establece contacto con el formando y le anima a continuar" enfrentándose a la apertura y expresión de ideas. Por consiguiente, se podría señalar que ésta es una herramienta poderosa para conducir trabajos en grupo y reuniones que buscan el máximo nivel de involucración y participación de los asistentes.

Figura 1. Comparación de la ubicación de las sillas entre el ciclo de aprendizaje experiencial y el método de aprendizaje tradicional.
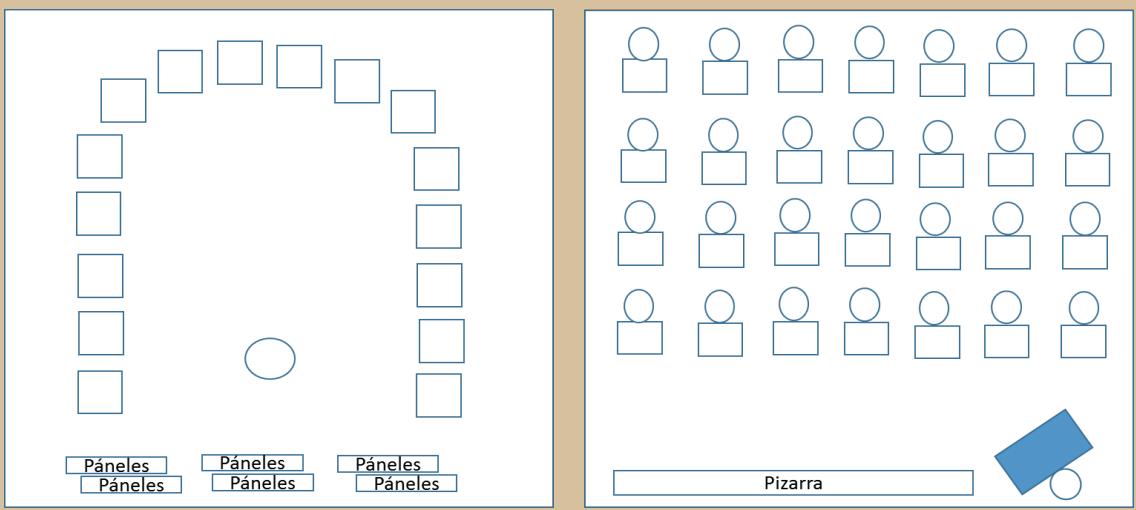

Fuente: elaboración propia con base en los dos métodos.

La técnica permite a los participantes aprender el uno del otro en un entorno que les permite conectarse entre sí. El metaplan también permite promover la conversación y producir comportamiento compartido basado en la experiencia, reflexión y acción. En últimas puede aumentar la dinámica grupal, el compartir mutuo de experiencias y por lo tanto desarrollar nuevos conceptos compartidos (Ataöv \& Ezgi Haliloglu Kahraman, 2009).

La tecnología de metaplan es bastante simple y al alcance de los recursos, pues se apoya así como lo señala EPISE (2007) en paneles, papelógrafo, papel de embalaje, tarjetas o fichas de cartulina, marcadores permanentes y mucha creatividad (véase imagen 1).

6 Como comúnmente se conoce a la técnica en Alemania. 


\section{Gingtites}

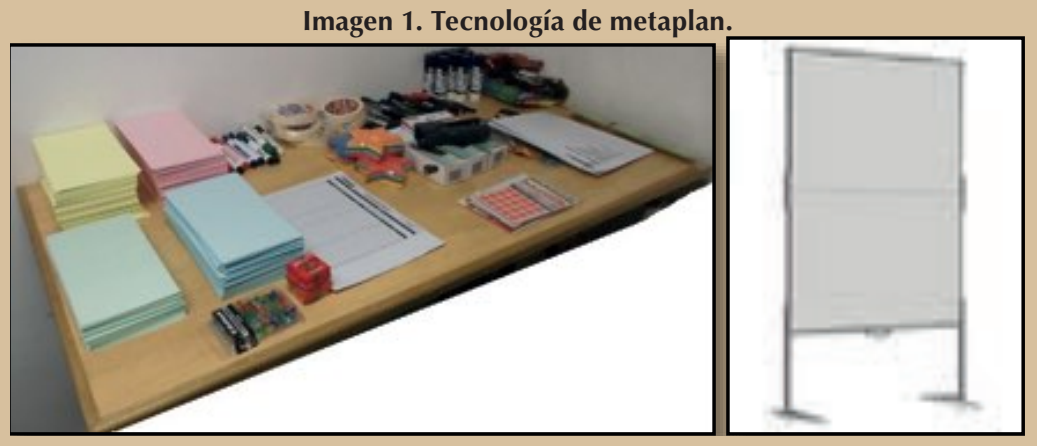

Fuente: material utilizado y adquirido por Esumer para impartir el seminario. El panel es una imagen tomada del portal de neuland, tienda especializada en el aprendizaje interactivo URL http://www.neuland-mallorca.com/

Los paneles son planchas o estructuras de aluminio hechos con corcho o porexpan. Sobre dichos paneles se prensan las tarjetas de cartulina. El papelográfo es una tabla que soporta pliegos de papel, sirve para consignar o publicar información delante del público objetivo. El papel de embalaje es utilizado para recubrir los paneles, sobre éste se pinchan las tarjetas o se pegan si se quieren conservar los resultados para exponerlos visualmente en el aula de clase. Las tarjetas o fichas de cartulina normalmente de tamaño $10 * 21 \mathrm{~cm}$ y de varios colores, se utilizan para escribir las ideas, temas o preguntas a trabajar. Y por último los marcadores, se usan con el fin de poder escribir y leer bien las ideas planteadas por los participantes y el facilitador.

La visualización en el aula de clase, es otro elemento, aparentemente de forma, pero preponderante como principio básico en la aplicación correcta del método así como de la técnica metaplan, en tanto el simple hecho de publicar visualmente los productos de cada clase o sesión de trabajo, facilita la remembranza y repaso de las ideas, las vivencias y los conceptos trabajados previamente.

\section{Aplicación práctica del método de aprendizaje experiencial y la técnica metaplan}

Como se ha mencionado, la Institución Universitaria Esumer impartió en el marco del proyecto de cooperación con la Agencia de cooperación alemana, GIZ, un seminario de formación a ejecutivos de pymes de tres sectores productivos, como ejecución del programa Global Business Exchange (GloBus). Dicho seminario realizado en agosto del presente año en la ciudad de Medellín, tuvo una duración de 30 horas.
El contenido del seminario se ciñó y estructuró bajo los requerimientos y propósitos del programa GloBus, por tal razón las instituciones aliadas fueron fieles al objetivo del programa el cual no era otro que posibilitar relaciones comerciales de cooperación con pymes alemanas. Igualmente para impartir el contenido temático propuesto, Esumer aplicó durante todo el seminario, el método de aprendizaje por experiencia y la técnica metaplan.

Los temas abordados durante el seminario fueron:

1. Entorno alemán: economía y sociedad alemana, formas de cooperación entre Colombia y Alemania, modalidades de inversión en Alemania, búsqueda de información sobre oportunidades en Alemania.

2. Habilidades de comunicación intercultural y negociación: ambiente cultural y de negocios en Alemania, estrategias de comunicación intercultural y estrategias de negociación internacional.

3. Análisis de mercado de Alemania y fortalecimiento de proyectos: formulación de objetivos, filtro de ideas de negocio y formulación de proyectos.

4. Estrategias de mercado internacional con Alemania: fundamentos de mercadeo, estrategia de precios y planeación estratégica de mercadeo. 
Los resultados obtenidos al finalizar el seminario por parte de los ejecutivos asistentes fueron altamente satisfactorios. Entre los resultados cualitativos se destacan los siguientes comentarios: "practicidad para obtener la información y generar el conocimiento", "el seminario fue muy ameno e interesante", "excelentes las actividades para ubicar en los procesos de negociación a los empresarios", "muy buena la metodología y el contenido, realmente aporta al crecimiento profesional","muy positivo" y "excelente".

Paralelamente, uno de los aspectos que más destacaron y ponderaron como positivos los asistentes, fue el alto nivel de motivación por el aprendizaje que imprime y permite el método, frente a este particular, sobresale el siguiente comentario: "agradezco por la información entregada, la motivación y entrega por parte del equipo de trabajo".

De los anteriores comentarios recibidos del seminario, se puede inferir que el método o ciclo de aprendizaje experiencial como instrumento en la enseñanza para adultos, realmente es útil, funcional y una herramienta poderosa para hacer llegar el conocimiento de manera práctica.

La evaluación del seminario también arrojó resultados cuantitativos, los cuales se aprecian en la siguiente tabla:
Tabla 1. Resultados de la evaluación realizada al seminario

\begin{tabular}{|c|c|c|c|c|c|c|c|c|c|}
\hline \multirow{2}{*}{ Aspecto a evaluar } & \multicolumn{3}{|c|}{$\begin{array}{l}\text { Resultado } \\
21 / 08 / 2014 \\
\end{array}$} & \multicolumn{3}{|c|}{$\begin{array}{c}\text { Resultado } \\
22 / 08 / 2014\end{array}$} & \multicolumn{3}{|c|}{$\begin{array}{l}\text { Resultado } \\
23 / 08 / 2014 \\
\end{array}$} \\
\hline & (;) & $\ominus$ & (2) & (-) & $\ominus$ & (2) & (:) & $\ominus$ & (2) \\
\hline $\begin{array}{l}\text { 1. Relevancia del } \\
\text { contenido }\end{array}$ & $100 \%$ & - & - & $100 \%$ & - & - & $100 \%$ & - & - \\
\hline $\begin{array}{l}\text { 2. Construyendo } \\
\text { conocimiento }\end{array}$ & $100 \%$ & - & - & $100 \%$ & - & - & $100 \%$ & - & - \\
\hline $\begin{array}{l}\text { 3.Calidad de la } \\
\text { presentación y } \\
\text { facilitación }\end{array}$ & $100 \%$ & - & - & $100 \%$ & - & - & $89 \%$ & $11 \%$ & - \\
\hline $\begin{array}{l}\text { 4.Aprendiendo de } \\
\text { los demás }\end{array}$ & $78 \%$ & $22 \%$ & - & $100 \%$ & - & - & $89 \%$ & $11 \%$ & - \\
\hline $\begin{array}{l}\text { 5.Personalmente } \\
\text { como se siente }\end{array}$ & $100 \%$ & - & - & $100 \%$ & - & - & $100 \%$ & - & - \\
\hline
\end{tabular}

* Las caritas indican satisfecho, medianamente satisfecho, no satisfecho.

Fuente: elaboración propia con base en los resultados de las evaluaciones aplicadas los días, 21, 22 y 23 de agosto de 2014.

De acuerdo con los porcentajes obtenidos en la tabla 1, se aprecia y corrobora las bondades del método de aprendizaje por experiencia. En los aspectos: 1) Relevancia del contenido y 2) Construyendo conocimiento la calificación fue $100 \%$ positiva lo que indica que los objetivos académicos del seminario se alcanzaron a cabalidad. En el aspecto 3) Calidad de la presentación y facilitación, también el resultado fue satisfactorio $100 \%$ en las dos primeras sesiones y $89 \%$ en la última sesión del seminario, porcentaje que igualmente es alto y positivo. En el aspecto 4) Aprendiendo de los demás, los resultados fueron igualmente satisfactorios en la primera sesión fue $78 \%$, en la segunda sesión fue $100 \%$ y en la tercera sesión fue de $89 \%$. En síntesis queda evidenciado que este método tiene cabida en los programas de formación y es una herramienta valiosa para impartir conocimiento donde la práctica y la experiencia es la gran protagonista. 


\section{Conclusiones}

La teoría de aprendizaje experiencial plantea un cambio significativo en la manera de concebir la educación y los procesos de aprendizaje. De esta perspectiva emergen enfoques muy diferentes sobre la forma en la que se debe llevar a cabo el proceso educativo, las relaciones apropiadas al momento de aprender e incluso la creación de conocimiento en sí.

Desde hace dos décadas ha habido un crecimiento importante en cursos basados en aprendizaje experiencial. Este crecimiento está apoyado en la gran cantidad de investigaciones que encuentran que estos cursos están asociados con una serie de resultados intra e interpersonales positivos que incluyen aspectos como el desarrollo moral, habilidades de liderazgo, y el aprecio de la diversidad tal como lo expone Abe (2011) citando a (Eyler \& Giles, 1999).

Son muchos las universidades, instituciones e incluso gobiernos que utilizan esta teoría con resultados positivos en diversas áreas. Por ejemplo en el trabajo de Ataöv \& Ezgi Haliloglu Kahraman (2009) se implementa la metodología para que a través de un trabajo en conjunto se fomenten acciones sociales. Los participantes en este programa lograron sus resultados a través de una serie de trabajos de grupo, sesiones de reflexión y discusiones plenarias. Usaron su experiencia para formular colectivamente un concepto compartido; reflexionaron sobre ella y se comprometieron a emprender acciones de mejora.

En este caso, la formación en aprendizaje experiencial se realizó gracias a un convenio entre la GIZ y la Institución Universitaria Esumer y luego de esta se aplicó a un grupo de empresarios de la ciudad de Medellín. Los resultados fueron satisfactorios tanto por la apropiación de conocimiento como con el resultado del seminario en sí cuyo producto fue la creación de un plan de negocios y de cooperación con Alemania.

Se pudo comprobar en la práctica, las bondades de esta teoría y se encuentra también el potencial de este método en la parte de crecimiento personal y profesional que se puede aplicar desde lo colectivo a comunidades y entornos profesionales o académicos. Por lo tanto la aplicación y las implicaciones del aprendizaje experiencial son innumerables.

\section{Referencias}

Abe, J. A. a. (2011). Positive emotions, emotional intelligence, and successful experiential learning. Personality and Individual Differences, 51(7), 817-822. doi:10.1016/j.paid.2011.07.004

Ataöv, A., \& Ezgi Halilo囚lu Kahraman, Z. (2009). Constructing collaborative processes through experiential learning: Participatory planning in Kaymaklı, Turkey. Habitat International, 33(4), 378-386. doi:10.1016/j.habitatint.2008.11.001

CEFE (1998). Manual para facilitadores, Gesellschaft für Technische Zusammenarbeit-GTZ-, Colonia.

Cliffs, E. (2006). Experiential learning : experience as the source of learning and development, (1984).

Dewey, J., 1938. Experience and Education Kappa Delta PI Lecture Series. Collier-Macmillan Books 1963, London.

EPISE (2007) Metaplan: sesiones formativas y reuniones de trabajo más efectivas consultado en URL https://sisconpes.dnp.gov.co/ LinkClick.aspx?fileticket=j/RV6ZIN4qA=

Fowler, J. (2008). Experiential learning and its facilitation. Nurse Education Today, 28(4), 427-33. doi:10.1016/j.nedt.2007.07.007

González, M. L., Marchueta, J., \& Vilche, E. (2013). Modelo de aprendizaje experiencial de Kolb aplicado a laboratorios virtuales en Ingeniería en Electrónica (p. 8).

Kolb, D. A., Boyatzis, R. E., \& Mainemelis, C. (1999). Experiential learning Theory: Previous Research and New Directions (p. 16).

Konak, A., Clark, T. K., \& Nasereddin, M. (2014). Using Kolb's Experiential Learning Cycle to improve student learning in virtual computer laboratories. Computers \& Education, 72, 11-22. doi:10.1016/j.compedu.2013.10.013

Management, G. E. De, \& Ansart, S. (2013). Accreditation Of Prior Experiential Learning, 9(4).

Manolis, C., Burns, D. J., Assudani, R., \& Chinta, R. (2013). Assessing experiential learning styles: A methodological reconstruction and validation of the Kolb Learning Style Inventory. Learning and Individual Differences, 23, 44-52. doi:10.1016/j.lindif.2012.10.009

Melchers, K. G., Shanks, D. R., \& Lachnit, H. (2008). Stimulus coding in human associative learning: flexible representations of parts and wholes. Behavioural Processes, 77(3), 413-27; discussion 451-3. doi:10.1016/j.beproc.2007.09.013

Metallidou, P., \& Platsidou, M. (2008). Kolb's Learning Style Inventory-1985: Validity issues and relations with metacognitive knowledge about problem-solving strategies. Learning and Individual Differences, 18(1), 114-119. doi:10.1016/j.lindif.2007.11.001

Mónica, L., \& Ernesto, A. (n.d.). Modelo de aprendizaje experiencial de Kolb aplicado a laboratorios virtuales en Ingeniería en Electrónica.

Nooghabi, S. N., Iravani, H., \& Fami, H. S. (2011). A study on present challenges on experiential learning of university students (University of Tehran, The Colleges of Agriculture and Natural Resources, Iran). Procedia - Social and Behavioral Sciences, 15, 3522-3530. doi:10.1016/j.sbspro.2011.04.329

Rone, T. R. (2010). Engaged Education : Experiential Learning, Intensive Field Experiences, and Social Change.

UNESCO (1993) La educación a distancia y la función tutorial, Oficina Subregional de Educación de la Unesco, San José de Costa Rica. 